\title{
The Relation between Prior Knowledge and Students' Collaborative Discovery Learning Processes
}

\author{
Hannie Gijlers, Ton de Jong \\ Department of Instructional Technology, University of Twente, P.O. Box 217, \\ 7500 AE Enschede, The Netherlands
}

Received 10 March 2004; Accepted 20 July 2004

\begin{abstract}
In this study we investigate how prior knowledge influences knowledge development during collaborative discovery learning. Fifteen dyads of students (pre-university education, 15-16 years old) worked on a discovery learning task in the physics field of kinematics. The (face-to-face) communication between students was recorded and the interaction with the environment was logged. Based on students' individual judgments of the truth-value and "testability" of a series of domain-specific propositions, a detailed description of the "knowledge configuration" for each dyad was created before they entered the learning environment. Qualitative analyses of two dialogues illustrated that prior knowledge influences the discovery learning processes, and knowledge development in a pair of students. Assessments of student and dyad definitional (domain-specific) knowledge, generic (mathematical and graph) knowledge, and generic (discovery) skills were related to the students' dialogue in different discovery learning processes. Results show that a high level of definitional prior knowledge is positively related to the proportion of communication regarding the interpretation of results. Heterogeneity with respect to generic prior knowledge was positively related to the number of utterances made in the discovery process categories "hypotheses generation" and "experimentation." Results of the qualitative analyses indicated that collaboration between extremely heterogeneous dyads is difficult when the high achiever is not willing to scaffold information and work in the low achiever's zone of proximal development. (C) 2005 Wiley Periodicals, Inc. J Res Sci Teach 42: 264-282, 2005
\end{abstract}

There is a large body of research on the effects of discovery learning (e.g., de Jong \& van Joolingen, 1998; Friedler, Nachmias, \& Linn, 1990; Reimann, 1991) and also on the effects of collaborative learning (Rochelle, 1992; Webb, 1991), but less attention has been given to the combination of collaboration and discovery. The majority of studies on scientific discovery learning focus on discovery learning by individuals and, as yet, only a few studies (Rochelle, 1992; Roth \& Roychoudhury, 1992; Teasley, 1995) have investigated the processes that occur when

Correspondence to: H. Gijlers; E-mail: gijlers@edte.untwente.nl DOI 10.1002/tea.20056

Published online 3 February 2005 in Wiley InterScience (www.interscience.wiley.com). 
students work together on a discovery learning task. Okada and Simon (1997) stressed that a model for collaborative discovery learning should take into consideration the prior knowledge and actions of all group members. In this study we introduce a model to describe the knowledge states of two collaborating students in a discovery learning setting. From this model we discuss the influence of prior knowledge on collaborative discovery learning.

Discovery learning encourages students to be active agents in their own learning process. Within a discovery learning environment the students' main task is to find the properties of a domain. These properties are not presented to the students in a direct manner, but are to be discovered through experimentation and interpretation. Two approaches can be identified in previous research on discovery learning. The first approach puts an emphasis on the discovery learning processes, the second approach focuses on knowledge development.

Discovery learning processes have been classified following different classification schemes (e.g., Friedler, Nachmias, \& Linn, 1990; Kuhn, Black, Keselman, \& Kaplan, 2000; Njoo \& de Jong, 1993; White, Shimoda, \& Frederiksen, 1999). In this study we use the classification scheme of Njoo and de Jong (1993), who made a distinction between transformative processes and regulative processes. Regulative processes refer to the control over the learning process. Examples of regulative processes include planning and monitoring. Transformative processes are processes that more or less directly generate new knowledge and comprise processes such as analysis (orientation), hypothesis generation, testing, (data) interpretation, and evaluation. During orientation, students search for information about the domain and the task at hand. They identify the variables and parameters in the model and indicate its general properties. Orientation can be done on the basis of the students' own prior knowledge, the group's prior knowledge (in a collaborative setting), additional information, and information available in the learning environment. Hypothesis generation can be seen as one of the central processes in discovery learning. In a hypothesis, students specify the relation between input and output variables. Experimentation refers to all activities that deal with the design and execution of experiments. Interpretation concerns activities that deal with the interpretation of data and results. Evaluation is seen as activities that allow students to reflect on their experiments and that put the results in a broader context.

Another approach in research on discovery learning describes the evolving knowledge. Klahr and Dunbar's (1988) SDDS (Scientific Discovery as Dual Search) model is an example of this approach. The SDDS model describes discovery learning as a search process through two spaces: the hypothesis space and the experiment space. The hypothesis space is the search space that contains all rules describing the phenomena that can be observed within the domain. The experiment space consists of all experiments that can be performed within the domain. To portray discovery learning in complex domains van Joolingen and de Jong (1997) extended the SDDS model. They introduced different regions in hypothesis space and designed a taxonomy to describe different search operations. Figure 1 shows a graphical overview of the different regions in the hypothesis space (based on van Joolingen and de Jong, 1997).

The universal hypothesis space contains all hypotheses that could possibly be stated. The learner hypothesis space is a subspace of the universal hypothesis space and contains all propositions, variables, and relations the learner knows of and that he or she could possibly use to describe the domain to be discovered. The learner domain space is a subset of the learner hypothesis space and represents the learner's knowledge concerning the domain and indicates the propositions the learner thinks are true or considers as possibly true in the domain. The target conceptual model describes the propositions that are valid in the domain and covers the knowledge to be discovered. During the discovery learning process the knowledge base of the learner changes. When students gain knowledge about the underlying model, their learner domain space 


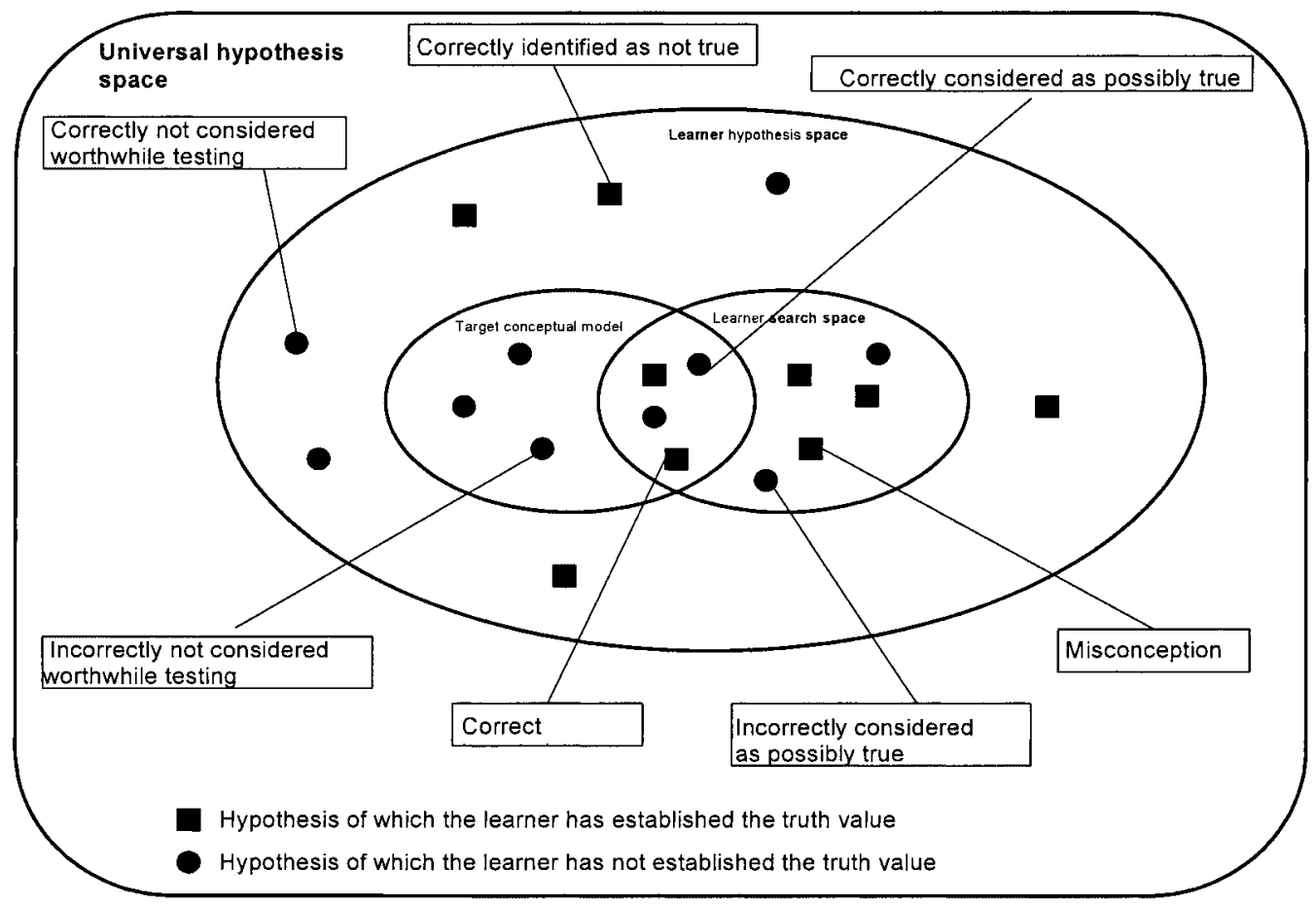

Figure 1. The extended SDDS model: a knowledge configuration with hypotheses included.

moves toward the target conceptual model. This implies that the extended SDDS model is a dynamic model that changes during the process of discovery learning. A student might enter the learning environment with a minimum of relevant knowledge. In this case the learner domain space and the target conceptual model have only a small amount of overlap. While the student gains knowledge through interaction with the environment, the learner space will move toward the target conceptual model. This also means that Figure 1 displays only one possible knowledge configuration, with, in this case, only slight overlap between the student's knowledge and considerations and the target conceptual model.

Within the extended SDDS model we distinguish propositions of which the student has already assessed the truth-value and propositions of which the truth-value is still unknown to the student. The propositions can be located in different regions of the extended SDDS model (Figure 1). The combination of the status of a truth-value of a certain proposition and its location in the SDDS model offers the possibility to describe a student's knowledge configuration. For example, when the truth-value of a certain proposition has not been established, and this proposition is located in the overlap between the target conceptual model and the learner domain space, this proposition is correctly considered as possibly true.

The extended SDDS model as presented in Figure 1 represents the knowledge configuration of a single student. Within a collaborative discovery learning setting two or more students interact with each other. This implies that there is feedback not only from the experimental outcomes, but also from a partner's prior knowledge. The alternative propositions stated by student A, are likely to influence the search of student B. A gap in the knowledge of student B might be filled in by knowledge from student A. In this way knowledge is co-constructed by the collaborating students. 


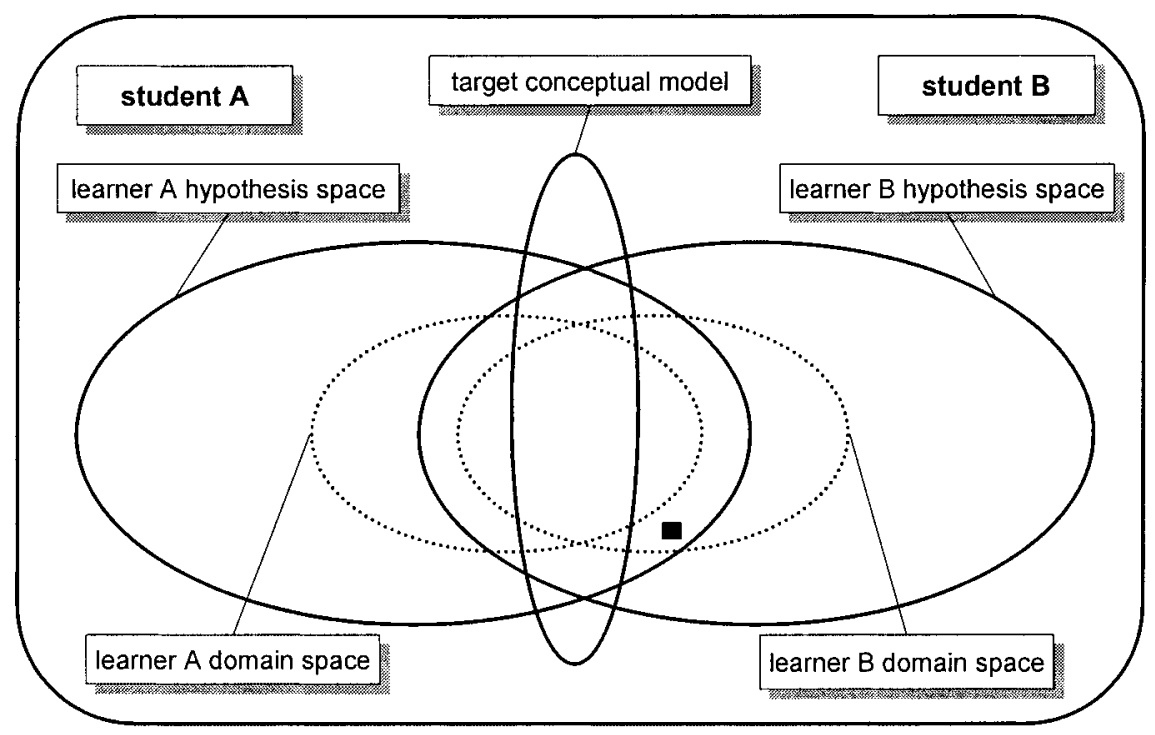

Figure 2. The extended SDDS model: an example of a knowledge configuration for two students.

The extended SDDS model as presented in Figure 1 can be adjusted to a model representing the knowledge configuration of two students working together on a scientific discovery learning task.

In the model presented in Figure 2, individual and shared knowledge can be distinguished. This distinction is important because not all knowledge and assumptions are shared. Propositions, variables, and relations that a student considers are represented by the learner domain space. When two students work together they might agree on some ideas and disagree on others. The overlap between the learner domain spaces of both students can be described as a common domain space. We can say that the overlap depicts the common ground of mutual understanding and shared assumptions that have been claimed to be necessary for many aspects of communication (Baker, Hansen, Joiner, \& Traum, 1999). The nonoverlapping parts display students' individual knowledge.

Different combinations of prior knowledge within a dyad might lead to different communication and learning processes. Imagine a dyad of students working within the physics domain of kinematics, such as with a simulation of the braking distance of a scooter. They consider the following statement: "If the velocity of a scooter is enlarged by a factor of two the braking distance of the scooter is also enlarged by a factor of two." Student A knows that this statement is not true, whereas Student B thinks the statement is true. In Figure 2 this is represented by the fact that the proposition (a black square) is located within the hypothesis space of student A but outside the learner domain space of student A and within the learner domain space of Student B. The proposition is not located within the target conceptual model, which indicates that the statement in this proposition is not true. In this case the first student might start explaining to student B that there is no direct positive linear relationship between scooter speed and braking distance. In this particular situation student B has the opportunity to learn something from student A. However, if both students had thought the proposition was true, it would become more difficult to resolve the misconception. If both students considered the hypothesis as possibly true but were not sure, this could encourage them to do an experiment. This example illustrates the potential influence of individual prior knowledge on discovery learning processes within a dyad. If the students have different opinions or prior 
knowledge this is of possible influence on their discovery learning process. Thus, in the aforementioned example, student A could scaffold the knowledge for student B.

Differences in prior knowledge and their influences on the learning process have been studied extensively in the literature (Vygotsky, 1962, 1978; Webb, 1991). Vygotsky (1978) discussed collaboration within the zone of proximal development. The zone of proximal development was described by Vygotsky as "the distance between the actual development level as determined by independent problem solving and the level of potential development as determined through problem solving under adult guidance or in collaboration with more capable peers" (Vygotsky, 1978, p. 86). This indicates that students can perform certain tasks under the supervision of a more capable person (e.g., an adult) that they cannot perform on their own. From Vygotsky's perspective, students have different roles during the learning process. The more capable peer guides the less capable peer during the learning process. A similar approach was advocated in the work by Newman, Griffin, and Cole (1989), who described collaboration as knowledge scaffolding by an expert and knowledge appropriation by the novice. Newman et al. (1989) further expounded on these concepts in their discussion of the construction zone. The construction zone is an interactive zone where students work together on problems that one of them could not solve individually. Cognitive change takes place within the construction zone. The supportive structure is determined not only by the support students receive from each other but also by the environment and the task structure.

Furthermore, a collaborative learning setting provides the students with the opportunity to discuss alternative hypothesis. According to Kneser and Ploetzner (2001) and Okada and Simon (1997), the discussion of alternative hypotheses is an important aspect of collaborative discovery. Okada and Simon (1997), for example, compared dyads and individuals working on a discovery learning task. They found that pairs were more successful. Additionally, pairs discussed and constructed more alternative hypotheses.

These alternatives may reveal individual misconceptions that become apparent through verbalization (Vahey, Enyedy, \& Gifford, 1999).

In the present study we explore collaborative discovery learning. To be more precise we focused on the influence of prior knowledge on the development of knowledge and the associated learning processes within a collaborative discovery learning context. In a discovery learning environment built around a simulation in the domain of kinematics, we investigated the knowledge development of dyads of students. We assessed prior knowledge by a number of assessment methods. A hypothesis test was developed to assess students' prior knowledge and beliefs about the hypothesis within the domain. More traditional tests were used to assess generic knowledge and definitional domain knowledge. Dyads of students worked together on a collaborative discovery learning task. Based on the test results and transcribed protocols, developing knowledge configurations were assessed.

\section{Method}

\section{The Domain}

The learning environment in this study concerned the physics domain of kinematics. The domain of kinematics is prone to misconceptions. The misconceptions of students are grounded in extensive personal experiences and instruction (Halloun \& Hestenes, 1985). Research has shown that students taking an introductory physics course, at a university level, still experience serious trouble with kinematics. Trowbridge and McDermott (1980) studied university students' reasoning about position, velocity, and acceleration. They found that, even after instruction, about $20 \%$ of the students still confused the concepts of speed and acceleration. 
Computer simulations can address some of the problems students' experience in the domain of kinematics. The animation of motion combined with a graph can help students understand kinematical graphs.

\section{The Learning Environment}

In the present study, students worked with a computer-based simulation environment in which the central part was a simulation on kinematics. The learning environment was developed with the SimQuest authoring environment (van Joolingen \& de Jong, 2003). More information on SimQuest and examples of applications developed with SimQuest can be found on the website (www.simquest.nl).

Students worked together in a face-to-face setting. Sharing a tool and a task created a natural need to communicate and share ideas. In the learning environment students manipulated values of input variables, and observed the behavior of output variables. Output was presented to the students in the form of animations, graphs, and numbers.

In order to guide the student during the learning process the learning environment contained instructional support. The full model of a simulation is often very complex. We used model progression (White \& Fredriksen, 1990) and divided the domain into four levels: an introductory level and three progression levels. The introductory level was developed to introduce learners to the learning environment. The model in the first level focused on initial velocity, acceleration, time, and final velocity $(\mathrm{v}(\mathrm{t})=\mathrm{v}(0)+\mathrm{a} \cdot \mathrm{t})$. The relevant variables were presented to the student one at a time. In the first progression level, students could test propositions such as: "If the acceleration of a car equals zero then the final velocity of this car will equal the initial velocity." Within the second progression level, the students worked with simulations on distance covered. In the third and fourth progression levels, the concepts of mass and friction were introduced to the students. After the introductory level learners were free to start at any level and move back and forth between them.

Assignments were used to guide students through the key elements of the simulation and provide them with short-term goals. Together with model progression, assignments disaggregated the complex model into smaller portions. In this learning environment we used different types of assignments. Some assignments asked students to find the relation between two or more variables (investigation assignments), other assignments asked the learners to explain a specified relation (explication assignments), and still other assignments asked students to predict the value of a variable under specified conditions (specification assignments). Figure 3 displays a simulation of a truck and an assignment. The (specification) assignment in Figure 3 is taken from the third progression level. The truck in the simulation starts from a standstill. Students are asked to predict the velocity of the truck after 11 seconds. They are provided with information about the mass of the motor truck and the trailer, friction from the road, friction from the air, and the driving force.

\section{Sample}

The sample consisted of 30 fourth year (15-16 years old) high school students ( 15 boys and 15 girls) in The Netherlands. All students were from the same school and followed a university preparation track. Prior to this study, students completed an introduction to kinematics in their regular physics classes. This introduction covered the definitional knowledge needed for working in the simulation environment. Subjects participated in the study on a voluntary basis and received a small reward for their participation. Subjects were randomly paired with another student. All subjects had sufficient computer experience. 


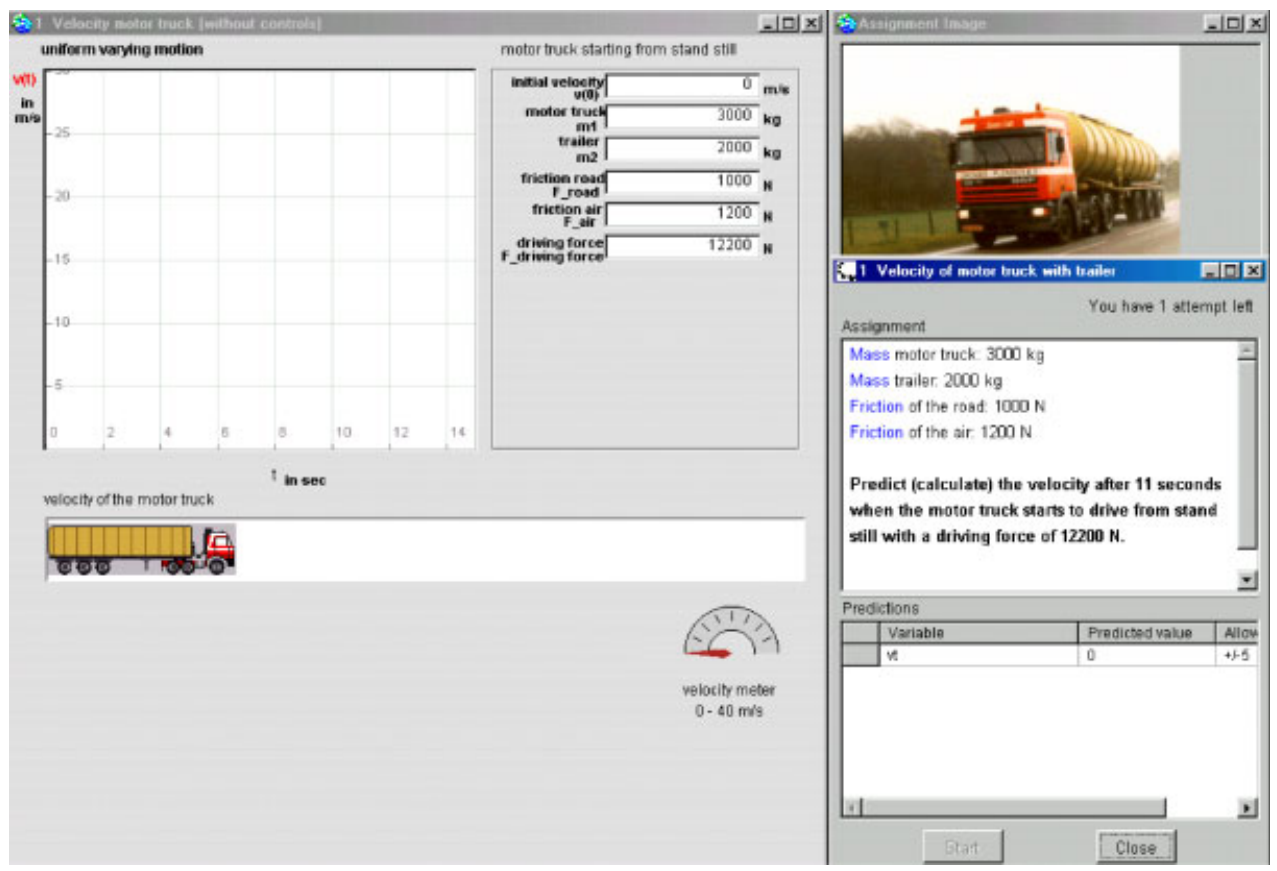

Figure 3. Screenshot of a simulation with an assignment.

\section{Knowledge Tests}

Different tests were used to assess the different kinds of prior knowledge and students' knowledge configurations. A definitional knowledge test was used to assess students' knowledge of concepts and variables in the domain. A generic knowledge test focused more on students' ability to work with mathematical relations, such as their ability to interpret graphs and experimental outcomes and their discovery skills. The TIPS II (Test of Integrated Process Skills II; Burns, Okey, \& Wise, 1985), TOGS (Test of Graphing in Science; McKenzie \& Padilla, 1986), and TUGK (Test of Understanding Graphs in Kinematics; Beichner, 1994) inspired our generic knowledge test. The propositions test focused on students' judgment of the truth-value of a set of domain-specific propositions. Copies of the test described in this study are available on request.

Definitional knowledge test. Swaak and de Jong (1996) defined definitional knowledge as declarative conceptual knowledge. The objective of the definitional knowledge test is to determine whether students know the concepts and variables that were relevant in the learning environment. In this study we tried to determine if students knew the concepts that are important in the domain of kinematics, such as acceleration, air friction, and velocity. The definitional knowledge test consisted of 22 multiple-choice items (with four answer alternatives). Of the 28 students from whom the data were analyzed there was an average score of $12.67(\mathrm{SD}=4.59)$. Cronbach's alpha for the test was 0.83 , which indicates high internal consistency.

Generic knowledge test. Generic knowledge can be defined as the knowledge needed to recognize and work with numerical and graphically depicted relations between two or more variables (Ploetzner \& Spada, 1992). Knowledge about relations is needed to state hypotheses about relations between variables and to interpret the results of experiments. In our study this implies that we have to assess whether or not a student possesses the knowledge and skills needed 
to understand qualitative and quantitative relations in a general way. The generic knowledge test consisted of 36 items. The average score of the 28 students from whom data were analyzed was 26.65 ( $\mathrm{SD}=5.61)$. All items were multiple-choice questions with four answer alternatives. The test was divided into three sections. The first section consisted of 13 items and was designed to assess students' knowledge about mathematical relations (Cronbach's alpha 0.74). The second section also consisted of 13 items and measured the ability to work with graphs (Cronbach's alpha 0.61). The third section consisted of 10 items and examined student performance in the areas of planning and conducting an investigation. Items in this section of the test aimed at the identification of relevant variables, the design of an experiment, the ability to state a hypothesis, and identification of data that support a hypothesis. For the generic knowledge test as a whole, a Cronbach's alpha of 0.85 was determined.

Cronbach's alpha for the combined test is higher than the alphas for the separate test sections. This can be explained by the fact that the combination of sections leads to a test with more items, and adding items to a test can have a positive influence on the reliability of a test (Brown, 1910; Spearman, 1910). The alpha of the generic skills section was quite low. A more detailed analysis of the items and the students' scores shows that the low alpha was due to a low variation between subjects. The sum of squares of the between-subject variation was 6.24 with 27 degrees of freedom.

Pearson correlations between the different test sections suggest that the three measures have a common basis. The Pearson correlation between the sections on discovery learning skills and mathematical skills was quite high $(r(28)=0.83, p<0.05)$; the Pearson correlation between discovery skills and graphing skills yielded $r(28)=0.56(p<0.05)$; and the correlation between the section on mathematical skills and graphing skills reached $r(28)=0.71(p<0.05)$.

The fact that the different sections were highly correlated, and that a combination of these sections resulted in a higher reliability, led us to combine the different sections into one test score expressing the generic knowledge of students.

The correlation of the average mark students received on the science test administered by their teacher (referred to as teacher's grading) and the definitional knowledge pretest was high $(r(28)=0.85, p<0.001)$. The same can be said about the correlation between the teacher's grading and students' scores on the generic knowledge test $(r(28)=0.86, p<0.001)$ and the correlation between the definitional and generic knowledge test $(r(28)=0.86, p<0.001)$.

No significant relations between scores on the tests (generic and the definitional knowledge test) and gender were found. This implies that there is no need to correct for these variables when examining the test scores.

Proposition test. To assess students' judgments of the truth-value of propositions in the domain a proposition test was developed. In this test the students were confronted with 26 different propositions. Along with each proposition three questions were asked. First, students had to decide whether they were familiar with the proposition. Second, students had to indicate whether they thought the proposition was true, possibly true, possibly false, or false. Finally, they had to decide if they considered the proposition worthwhile to test. The answers given to these three questions were used to determine the configuration of the extended SDDS model for each student. The propositions test was administered as a paper-and-pencil test.

\section{Communication and Interaction}

During the learning session students communicated face to face. For this study we used the verbal interactions between students as a window on the students' discovery learning processes (Webb, 1991). All verbal interaction was recorded, transcribed, and coded in a stepwise manner. All actions that students performed within the learning environment were logged. From the log- 
files we derived information about the time students spent at a certain level and the type and number of assignments they made.

\section{Coding and Scoring}

The transcribed protocols were coded in a stepwise manner. The coding scheme was based on the different discovery learning processes distinguished by Njoo and de Jong (1993). First, all the dialogues were segmented into utterances. An utterance is defined as a series of words with a single communicative function; it is a distinct message from one student to another or from the student to him- or herself. Second, each utterance was categorized as on- or off-task communication. Offtask communication was not further categorized. Third, on-task communication was further categorized as technical, regulative, or transformative. All utterances related to technical features of the learning environment, such as closing and opening an assignment or window, were coded as technical. Utterances related to planning or monitoring of the learning process were coded as regulative. Communication that directly yielded knowledge was coded as transformative. Fourth, all communication referred to as transformative was further analyzed. As indicated earlier we distinguished the following transformative processes: orientation; hypothesis generation; experimentation; and interpretation.

A second coder was trained to work with the coding system and coded $10 \%$ of the data independently from the first coder. The interrater reliability coefficients of coding utterances in terms of on- and off-task communication reached 0.95 (Cohen's kappa). Interrater reliability of coding utterances in terms of technical, regulative, and transformative communication reached 0.90 (Cohen's kappa), and the interrater reliability regarding the transfomative processes reached 0.68 (Cohen's kappa). The findings presented in the Results section are based on the coding of the first coder.

\section{Procedure}

In the week before the learning sessions the students took the generic and definitional knowledge pretests during their physics lessons. The study was conducted over 15 sessions. Each session consisted of the withdrawal of two dyads from their regular physics class to participate in this study. Students were asked to complete the proposition pretest and received a summary of the introduction. Subsequently, students entered the introductory level of the simulation to get used to the environment. After approximately 7 minutes the students were asked to leave the introductory level and start working on the learning task. Each dyad participated in one 50-minute block of interaction with the learning environment. During the session students were provided with a short description of the program on paper. The description covered the issues addressed during the introduction. Throughout the session the students were free to talk to their partner. Furthermore, students were allowed to use a calculator and make notes during the experiment. Due to technical difficulties one of the dyads was not able to complete the entire learning session. The results of this dyad are not detailed in the Results section.

\section{Results}

First, we provide a brief overview of students' communication. Second, we report on the relations between the knowledge tests and students' communication regarding discovery learning processes. Finally, we use excerpts from the students' dialogues to highlight students' development of knowledge configurations during the collaborative discovery learning session. 


\section{Analysis of Communication}

During the learning sessions, we recorded all verbal interactions; 28 students in 14 dyads made a total number of 4358 utterances. The number of utterances dyads made ranged from a minimum of 138 utterances to a maximum of 486 utterances. Protocol analyses revealed that utterances of each partner in a dyad were fairly equitable with an average of $47.8-52.2 \%$ $(\mathrm{SD}=1.67)$.

The fact that both members of a dyad contributed an almost equal number of utterances to the dialogue in combination with the qualitative analysis of the protocols suggests that turn-taking was present during the collaborative discovery learning process.

Table 1 provides an overview of on-task and off-task communication and the different categories of on-task communication. The percentage of off-task communication was $6.7 \%$, which can be considered low. Examination of the protocols showed that off-task communication mostly appeared at the beginning and end of a learning session. Examples of off-task communication are statements such as giving compliments and school-related topics such as the lesson plan. Of all utterances, $2.1 \%$ related to technical problems students experienced in the environment.

All remarks related to planning or monitoring were coded as regulative. About $16 \%$ of all remarks made by the students could be called regulative. Most of these remarks occurred at the beginning of the session after the students had introduced themselves, and when they had to choose a new assignment or experiment. Other regulative remarks were made after students had experienced (technical) problems or made some off-task remarks. It seemed that technical problems as well as off-task talking disturbed the discovery learning process and that the students had to recover from that disturbance by (re)stating their plans.

Table 1 shows that $74.8 \%$ of the on-task communication was related to transformative processes. All utterances directly related to the domain of the learning environment, the experiment, assignment, or problem within the environment that the student worked on were coded as a transformative process.

\section{Learning Processes}

Students' transformative communication was analyzed in terms of the discovery learning processes. We distinguished four processes based on a model designed by Njoo and de Jong (1993). The processes distinguished are orientation, hypothesis generation, experimentation, and interpretation. A detailed description of these processes was given earlier. Table 2 indicates that the majority of transformative utterances were related to orientation. This means that students made many exploratory remarks about the domain and the learning environment. It seems logical that a large percentage of utterances were devoted to orientation. Especially when students began

Table 1

Overview of types of communication in frequencies and percentages

\begin{tabular}{llccr}
\hline Category & Subcategory & Frequency & Percentage & Standard Deviation \\
\hline Off-task & & 292 & $6.7 \%$ & 8.08 \\
On-task & Technical & 91 & $2.1 \%$ & 2.20 \\
& Regulative & 716 & $16.4 \%$ & 12.05 \\
Total & Transformative & 3259 & $74.8 \%$ & 41.01 \\
\hline
\end{tabular}

Note: $N=14$ dyads. 
Table 2

\begin{tabular}{lccc}
\multicolumn{4}{l}{ Frequencies, percentages and standard deviations of transformative processes } \\
\hline Learning Process & Frequency & $\begin{array}{c}\text { Percentage of } \\
\text { Transformative }\end{array}$ & $\begin{array}{c}\text { Standard } \\
\text { Deviation }\end{array}$ \\
\hline Orientation & 2285 & 70.1 & 26.33 \\
Hypothesis & 39 & 1.2 & 2.69 \\
Experiment & 556 & 17.1 & 10.62 \\
Interpretation & 379 & 11.6 & 8.84 \\
Total & 3259 & & \\
\hline
\end{tabular}

working with the learning environment they felt the need to explore the possibilities of the environment and shared ideas about it. Only a small number of utterances were related to hypothesis generation.

\section{Prior Knowledge Interaction and Knowledge Construction}

In order to investigate the influence of prior knowledge on the students discovery learning behavior we computed the partial correlation (controlling for the total number of utterances) between the number of on-task, off-task, regulative, and transformative utterances of individual students with their scores on the different prior knowledge tests. We found significant negative correlations between the amount of technical utterances made by the students and their scores on the definitional knowledge test $(r(28)=-0.50, p<0.05)$, the generic knowledge test $(r(28)=-0.57, p<0.05)$, and the combination of tests $(r(28)=-0.56, p<0.05)$. The results suggest that students with lower pretest scores made more remarks about the technical aspects of the simulation. The majority of technical remarks referred to problems students experienced using the learning environment. Roth, Woszczyna, and Smith (1996) described that operating the software can distract students from the content that they are supposed to learn. The results of our study suggest that low-achieving students experienced more trouble operating the system than high achievers. However, the percentage of utterances regarding technical issues was still relatively small ( $2.1 \%$ of the total number of utterances; see Table 2 ).

Utterances related to transformative processes were further classified in terms of the following processes: orientation; hypothesis; experiment; and interpretation. To examine the relation between prior knowledge and the number of utterances that were coded into one of the transformative processes, we used a partial correlation controlling for the total number of utterances.

We found a significant correlation $(r(28)=0.34, p<0.05)$ between scores on the definitional prior knowledge test and the amount of communication related to interpretation of data. Other correlations were not significant. The results might indicate that definitional knowledge helped students to make sense of experimental outcomes.

We further investigated the influence of prior knowledge, from the perspective of differences in prior knowledge between the two students in a dyad. This means that the results presented focus on the scores and performances of dyads instead of individual students. For each dyad, we calculated the "score" difference between the partners on the generic knowledge test, the definitional knowledge test, and the combination of these two tests. The score difference of one pair of students differed by more than 2 standard deviations from the average score difference. The pair consisted of an extreme high-achieving and an extreme low-achieving student. This pair of students was considered as on outlier and their score difference was excluded from further analyses, leaving 13 dyads in the analyses. However, in the qualitative analysis, we take a closer look at the learning session of this particular dyad. The score differences were correlated with the 
number of utterances coded as one of the transformative processes, corrected for the total number of utterances. We found positive and significant relations between the score difference on the generic knowledge test and the number of utterances dyads made related to hypothesis generation $(r(13)=0.58, p<0.05)$ and the design and execution of experiments $(r(13)=0.67, p<0.05)$. Dyads consisting of students with a different level of generic prior knowledge produced more utterances related to hypothesis generation and to experimentation. Furthermore, positive relations were found between the amount of utterances related to experimentation and the score difference on the definitional test $(r(13)=0.70, p<0.05)$ and the combination of both tests $(r(13)=0.78, p<0.05)$. This suggests that heterogeneity supports talk about hypotheses and experiments. The fact that the score difference on the generic prior knowledge test and hypothesis generation are positively correlated suggests that heterogeneity with respect to generic skills stimulates the generation of hypotheses. The close to significant negative relation of the score difference on the generic knowledge test and the number of utterances made in the orientation category suggests that dyads that are more homogeneous with respect to generic skills will perform more orientating processes. Examination of the protocols showed that these dyads searched for extra information on variables and relations in the simulation environment.

Examination of the test scores showed that almost all homogeneous dyads consisted of students with low or average scores on the prior knowledge test. No homogeneous high-achieving dyads participated in the experiment. Combined with the negative correlation between orientation and generic skills and the significant positive correlation between hypothesis generation and experimentation this might indicate that low- and average-achieving students in homogeneous dyads share the same limited resources that keep these dyads from stating hypotheses and experimenting.

\section{Behavior Within the Simulation}

Log-files provided us with information about the dyads' behavior. We used the number of simulation runs and the number of assignments dyads completed successfully as an indicator of their behavior in the simulation. During the learning session each dyad shared a computer. The number of assignments students completed successfully was positively correlated with the score difference between partners' scores on the definitional knowledge test $(r(13)=0.62, p<0.05)$ and the combination of definitional knowledge and generic knowledge $(r(13)=0.60, p<0.05)$.

One could argue that within a heterogeneous dyad the higher achieving student could be the one solving the problem on his or her own. However, we found that, in our sample, both partners contributed about the same amount of utterances to a dialogue. This and the fact that the proportion of off-task utterances was rather small suggest that both partners were involved in on-task behavior.

\section{Discovery Learning Processes and Knowledge Development}

In this section, two excerpts from protocols are used to describe the students' knowledge development. The knowledge development of the two dyads is described in terms of the extended SDDS model. The initial knowledge configurations are based on students' scores on the prior knowledge tests (definitional, generic, and hypothesis).

In the first excerpt the dialogue of two average-achieving students (Judith and Simone) is analyzed. The prior knowledge base of Judith and Simone is similar. In the second excerpt we take a closer look at the dialogue between Inge and Arjan. Inge performed very well on the pretests and Arjan scored very low. Because of the large difference between the pretest scores of Inge and 
Arjan, their score difference was not statically analyzed. In the following subsection we explore their collaborative discovery learning process.

\section{Judith and Simone}

In this situation Judith and Simone are working on the second level of the simulation. The central formula within this level of the simulation environment is $\mathrm{s}(\mathrm{t})=\mathrm{v}(0) \cdot \mathrm{t}+\frac{1}{2} \mathrm{a} \cdot \mathrm{t}^{2}$. Judith and Simone are both average achievers according to their test results. Their learner domain spaces have a little overlap with the target conceptual model. Simone and Judith have some prior knowledge in common and share a few misconceptions. Their score difference (combination generic and domain) was 4 points.

Before the episode transcribed in Table 3, Simone and Judith worked on the following problem: "What is the acceleration of your moped when you cover a distance of 25 meters in

Table 3

Episode from the transcribed discourse of Judith and Simone

\begin{tabular}{|c|c|c|}
\hline Turn & Name & Transcribed Discourse \\
\hline 1 & $\mathrm{~J}$ & I don't get this one either. \\
\hline 2 & $\mathrm{~J}$ & What about acceleration, this formula. \\
\hline 3 & $\mathrm{~S}$ & Has nothing to do with this. \\
\hline 4 & $\mathrm{~J}$ & Do we have to do something with a formula? \\
\hline 5 & $\mathrm{~S}$ & I don't get this one either. \\
\hline 6 & $\mathrm{~J}$ & We can't solve it. \\
\hline 7 & $\mathrm{~J}$ & We can't spent al our time on this problem. \\
\hline 8 & $\mathrm{~J}$ & Let's go to level 3 . \\
\hline 9 & $\mathrm{~S}$ & No this was hard enough, already. \\
\hline 10 & $\mathrm{~S}$ & Let's do one over here. \\
\hline 11 & $\mathrm{~S}$ & Estimate the distance covered. \\
\hline 12 & $\mathrm{~J}$ & Moped is starting from standstill. \\
\hline 13 & $\mathrm{~J}$ & After 10 seconds. \\
\hline 14 & $\mathrm{~S}$ & It has covered 5 meters. \\
\hline 15 & $\mathrm{~J}$ & I think we start with 0.5 . \\
\hline 16 & $\mathrm{~S}$ & Formula? \\
\hline 17 & $\mathrm{~J}$ & $\mathrm{~s}(\mathrm{t})=0.5 \cdot 0.5 \mathrm{a} \cdot \mathrm{t}^{2}$ \\
\hline 18 & $\mathrm{~S}$ & Is 25 . \\
\hline 19 & $\mathrm{~S}$ & Put the answer in there and press enter. \\
\hline 20 & $\mathrm{~J}$ & Where? \\
\hline 21 & $\mathrm{~S}$ & Predicted value, over here. \\
\hline 22 & $\mathrm{~S}$ & Press enter. \\
\hline 23 & $\mathrm{~J}$ & The moped actually covers the .... \\
\hline 24 & $\mathrm{~S}$ & It's okay. \\
\hline 25 & $\mathrm{~J}$ & Yeah, and the formula is over there, also. \\
\hline 26 & $\mathrm{~S}$ & Let's give the other one a try. \\
\hline 27 & $\mathrm{~S}$ & Using that $\mathrm{s}(\mathrm{t})$ formula. \\
\hline 28 & $\mathrm{~S}$ & Well it's a $\mathrm{s}(\mathrm{t})$ diagram. \\
\hline 29 & $\mathrm{~J}$ & 25 meters is the distance covered, we were using a $v(t)$ formula all the time. \\
\hline 30 & $\mathrm{~S}$ & $\mathrm{~s}(\mathrm{t})=0.5 \cdot \mathrm{a} \cdot \mathrm{t}^{2}$ \\
\hline 31 & $\mathrm{~J}$ & Than the acceleration should be 2 meters. \\
\hline 32 & $\mathrm{~S}$ & Look that formula was also used in the estimation assignment. \\
\hline 33 & $\mathrm{~S}$ & It must be time or something? \\
\hline 34 & $\mathrm{~S}$ & Yeah we have to close. \\
\hline 35 & $\mathrm{~J}$ & We get something. \\
\hline
\end{tabular}


5 seconds and start from a standstill. Calculate the acceleration." Simone and Judith tried solving this problem without using a formula for distance covered. They did not succeed. We can say that Simone and Judith both lacked the knowledge needed to solve the assignment.

Simone and Judith both do not know that they should have used a formula that includes the distance covered. They make another attempt and start by looking up formulas within the program. In turns $1,2,3$, and 4, Simone and Judith try to find additional information in the learning environment, because they both do not know how to solve the problem assigned.

Simone asks what the formula with "half a" in it means. This actually is the formula the students have to use to solve this problem. Both Simone and Judith do not recognize the formula as a relevant formula for the present assignment (see turns 2, 3, and 4). They know of the existence of the formula but they do not consider it relevant for the assignment they are working on. This implies that this formula is located within the hypothesis space of both students but outside their domain spaces. Simone and Judith decide that they have spent enough time on this particular assignment and start a new assignment. In this assignment they have to estimate the covered distance of a moped. In the assignment they use a formula including covered distance and are able to solve the problem. They get back to the former problem and Simone suggests that they should use the $s(t)$ formula instead of the $v(t)$ formulas they have used before. She strengthens her suggestion by referring to the $s(t)$ diagram that is used in the simulation (turns 27 and 28). The formula becomes relevant to Simone and thus becomes part of her learner domain space. Judith notices that the 25 meters in the assignment actually refer to the distance covered and now she also thinks it makes sense to use the following formula: $\mathrm{s}(\mathrm{t})=0.5 \cdot \frac{1}{2} \mathrm{a} \cdot \mathrm{t}^{2}$ and she adds another reason as shown in turn 29. Because Judith is also considering the formula it also becomes part of her learner domain space.

Within the dialogue between Simone and Judith we can distinguish different phases. The prior knowledge of Simone and Judith regarding the assignment they are doing is quite similar. Therefore, the partners are not likely to assist each other with explanations or additional information. Earlier, we presented data suggesting that students with a similar knowledge base spent a higher proportion of their communication on orientation. New information must be obtained from another source. Simone and Judith start looking in the learning environment for a formula that might give them a clue. They do not recognize the relevant formula and decide to make another assignment (regulative process). In turn 15 they start an experiment. The results of this experiment influence their behavior. They decide to go back to the other assignment. Simone relates the results of the previous assignment to the present assignment and suggests that they should use the formula for covered distance. She is presenting a new idea to her partner. They decide to perform the experiment and discuss the results. The results confirm Simone's suggestions.

In this excerpt Simone and Judith use the environment as reference material. In turn 17, Judith refers to a formula she found in the simulation environment. In turn 23 Judith refers to the simulation. The animated moped is moving and the graph is changing. Simone (turn 28) is talking about the diagram. Judith and Simone use the environment to solve problems and create meaning. Sharing tables, graphs, and other reference material can promote learning. Moschkovich (1996) described the peer discussion of students talking about the lines on a computer screen. She finds that sharing references materials like the graphs on the computer is an important aspect of the negotiation of meaning. If we look from the perspective of Newman, Griffin, and Cole (1989), students can not only support each other, but tools can also be part of the supportive structure. A simulation can assist students' interaction during science learning (Roth, 1995). In the case of Judith and Simone, the simulation and the graph illustrate the phenomena under discussion, and the students refer to the graph in their reasoning process. 
Inge and Arjan

In Table 4 the conversation between Arjan and Inge is transcribed. The score difference between Inge and Arjans' pretest scores is rather large. Inge and Arjan were excluded from the quantitative analyses because their score difference was more than 2 standard deviations from the mean. They differed 26 points from each other (generic and domain). Inge performed very well on the test. Her learner domain space has a lot of overlaps with the target conceptual model. Arjan scored low on the pretest. His learner domain space has only a slight overlap with the target conceptual model.

Arjan and Inge have to calculate the acceleration of a motorbike. The motorbike was traveling at a velocity of 108 kilometers per hour and has to reach standstill in 90 meters to avoid a collision. If the motorbike slows down too quickly the bike will skid, but if it does not brake hard enough it will collide. Inge and Arjan are already working together for 20 minutes when they open this assignment. Most of the time Inge is calculating and explaining the assignments to Arjan. Arjan

Table 4

Episode from the transcribed discourse of Inge and Arjan

\begin{tabular}{|c|c|c|}
\hline Turn & Name & Transcribed Discourse \\
\hline 1 & I & Let's go to the next assignment. \\
\hline 2 & A & That skidding assignment is cool, don't you agree? \\
\hline 3 & I & Initial speed is 30 . Look they filled it in, over here. \\
\hline 4 & A & Well we have 90 meters. \\
\hline 5 & I & Yeah and we are driving 108 kilometers an hour. \\
\hline 6 & A & So what are we supposed to do next? \\
\hline 7 & I & Brake. \\
\hline 8 & I & The acceleration must be negative. \\
\hline 9 & I & We have to calculate the acceleration. \\
\hline 10 & A & So? \\
\hline 11 & I & Maybe we can start with the same formula we used before. \\
\hline 12 & I & $\begin{array}{l}\text { You have } 90 \text { meters, so that could be your covered distance; } 108 \text { kilometers } \\
\text { an hour equals } 30 \text { meter a second. We don't know the time. }\end{array}$ \\
\hline 13 & A & Well fine, we still only know three out of the six terms in the formula. \\
\hline 14 & I & Well maybe we can find these terms. \\
\hline 15 & A & Give it try. \\
\hline 16 & I & We have to find it. \\
\hline 17 & A & Fine. \\
\hline 18 & I & Let's take a look at this. \\
\hline 19 & I & The final speed has to be zero, because you have to reach standstill. \\
\hline 20 & I & That means we have a final speed of zero $\mathrm{v}(\mathrm{t})$ equals zero. \\
\hline 21 & I & Shall we continue calling 30 the initial speed? \\
\hline 22 & A & Fine with me. \\
\hline 23 & I & Final speed is zero and initial speed 30 , plus acceleration times $t$. \\
\hline 24 & I & I think that $t$ equals 30 divided by acceleration. \\
\hline 25 & A & So that helps, us a lot. [Sarcastic] \\
\hline 26 & I & Sstt, let me try. \\
\hline 27 & I & Here we go, $90=30+30 / a+5 \cdot a(30 / a)$ \\
\hline 28 & I & Let's calculate. \\
\hline 29 & A & Gee, what a fuss. \\
\hline 30 & A & We really are supposed to think. \\
\hline 31 & I & I think it should be -5 \\
\hline 32 & A & Try it? \\
\hline 33 & A & It's correct. \\
\hline 34 & I & Gee, you're the greatest. \\
\hline
\end{tabular}


finds it difficult to understand her reasoning. In the first level he is able to understand the major part of Inge's explanations, but now he is experiencing serious problems.

At the beginning it is not exactly clear to Inge how she should calculate the right answer, but she knows a lot of concepts and two formulas that she can use (turns 12, 13, 14, and 27); she decides to try (turn 28) them. All the formulas, relations, and concepts Inge needs to solve this problem are located within her learner domain space. Arjan does not share the large knowledge base that Inge has. He does not know exactly when to use a certain formula and is insecure about the meaning of concepts. This is reflected in the conversation. Arjan does not ask questions and does not contribute to the learning process anymore. His job is to click and double click. He agrees with Inge's decisions and expresses feelings of incapacity and indifference.

Inge's learner domain space and the target conceptual model almost completely overlap, in contrast to Arjan's learner domain space that is much smaller and covers only part of the target conceptual model. Nevertheless, the procedure Inge needs to solve the assignment is not located within her learner domain space at this moment, However, Inge is familiar with the relations, variables, and formulas that she needs to solve the problem. These are located within her hypothesis space. Inge uses her prior knowledge about the domain to tackle this new problem. She succeeds. She has learned to use the combination of the formulas, $\mathrm{s}(\mathrm{t})=\mathrm{v}(0) \cdot \mathrm{t}+0.5 \cdot \mathrm{a} \cdot \mathrm{t}^{2}$ and $\mathrm{v}(\mathrm{t})=\mathrm{v}(0)+\mathrm{a} \cdot \mathrm{t}$, to solve the problem and determine the missing variables. The procedure becomes a part of Inge's learner domain space. Unfortunately, Arjan has less prior knowledge about the domain. He knows the two formulas stated before, but experiences difficulties when he has to apply them. As a result of some remarks that Arjan makes during the rest of the session there are reasons to believe that he still has difficulties with the application of formulas and that the procedure did not become part of his learner domain space. After the learning session, the procedure has become part of Inge's learner domain space. Arjan's learner domain space still does not include the procedure, but it has become part of his hypothesis space. This means that he now knows that a procedure like this exists but that he is not able to apply it.

The protocol of Inge and Arjan illustrates that roles are changing during the collaborative discovery learning process. In the episode transcribed in Table 4 Arjan has serious trouble following Inge's actions and explanations. Arjan still communicates with Inge but his utterances do not contribute to the transformative processes anymore. In the beginning of the same session Arjan was able to follow Inge's reasoning and made some assignments himself. During the learning session, the assignments got more complex and, in contrast to Inge, Arjan was not able to handle this complexity.

In this case the proposition under discussion is located in the learner hypothesis space of the more capable peer and located outside the domain space and learner hypothesis space of the less capable peer. To assist Arjan, a major tutoring task is awaiting Inge.

In this situation the less capable peer (Arjan) is not familiar with the variables and relations Inge uses. We could say that, in this case, the proposition is probably not in the zone of proximal development of Arjan. The differences in prior knowledge between the two collaborating students are too large.

\section{Discussion}

In this study we have examined the relation between prior knowledge and collaborative discovery learning for dyads of students working together within the same learning environment. This is a potentially interesting learning scenario because the knowledge of the collaborating students can exceed the knowledge of both individual students (e.g., Dillenbourg, 1999). Collaboration offers possibilities for co-construction of knowledge; comparison of alternative 
viewpoints; and explication of plans, concepts, and ideas. Explication of ideas can induce cognitive conflicts that might facilitate cognitive change (Chan, 2001).

The results of this study suggest that group composition in terms of prior knowledge is related to discovery learning processes. Our results show that heterogeneous pairs talked relatively more about different hypotheses and the carrying out of experiments. The conversation of the more homogeneous low- and average-achieving dyads is more related to orientation processes. These pairs of students try to fill in their knowledge gaps by orientation in the environment. Homogeneous groups with low- or average-achieving students who share the same limited knowledge resources and misconceptions are more likely to experience difficulties creating a meaningful conversation and constructing new knowledge.

In a heterogeneous group the student with more prior knowledge can serve as a guide for the less capable peer. The low achiever learns from the explanations given by the high achiever and high achievers have to restructure their knowledge in order to give appropriate help. This restructuring helps the explainer to understand the material better. From this perspective, heterogeneous grouping is beneficial for both high- and low-achieving students (Webb, Welner, \& Zuniga, 2001).

However, the qualitative analysis of Inge and Arjan in our study suggests that extreme knowledge differences within a dyad lead to frustrating situations. At the end of the learning session Inge was solving the problems by herself and had stopped explaining the problems to Arjan. The complexity of the problems Inge tackles at the end of the learning session was quite high. The concepts and formula's Inge used to solve the problem were quite new or even unfamiliar to Arjan. He did not understand Inge's reasoning. In the case of Inge and Arjan we can observe that Inge was not functioning in Arjan's zone of proximal development and did not respond to the problems he was experiencing. Webb et al. (2001) distinguished between highability students who perform low in heterogeneous groups and high-ability students who perform high in heterogeneous groups. High-ability students that perform low in heterogeneous groups do not respond to help-seeking group members with elaborated explanations. For example, they might solve the problem without providing further information, or even insult the person who is seeking help. In a well-functioning group, low-ability students might actively search for help, and benefit from explanations by applying them to a similar problem.

Extra information about the domain is important for both homogeneous low- or averageachieving dyads and dyads with extreme differences between the students. In the case of Inge and Arjan it became clear that Arjan lacked domain knowledge, and could not keep up with Inge. When using collaborative discovery in a classroom context it is important that teachers observe the group processes and intervene when necessary; for example, by providing hints or pairing students in different groups.

Collaborative discovery learning can offer some unique opportunities for learning. Interaction with the simulation environment allows students to experiment within the phenomena in the domain and discuss these with their partner. Simulations can be coordinated with other instructional activities. For example, experiences within the environment, and elements of the discussion between peers, can be discussed in the classroom. Furthermore, observation of students' behavior in the collaborative discovery learning setting can provide teachers with valuable information on students' ideas and misconceptions in the domain.

The present study indicates that it is important for students to become aware of and discuss the differences between their own beliefs, concepts, or theories, and new information. Collaboration is one way to confront students with the beliefs of others. However, students in a collaborative discovery learning setting are not always aware of their knowledge gaps and initial differences. de Vries, Lund, and Baker (2002) argued that computer learning environments should be carefully engineered to 
stimulate discussion and provide the opportunity to support and guide students' activities and communication. One way to take this into account would be to redesign the learning environment in such a way that students with initial differences become aware of each others beliefs-for example, by assessing students' individual opinions about a number of propositions in the domain and combining them into a shared table that visualizes the opinions of both partners. The externalization of students' individual opinions might stimulate the discussion of alternative conceptions, which might lead to the refinement of students' knowledge and, eventually, cognitive change.

\section{References}

Baker, M., Hansen, T., Joiner, R., \& Traum, D. (1999). The role of grounding in collaborative learning tasks. In P. Dillenbourg (Ed.), Collaborative learning. Cognitive and computational approaches (pp. 31-63). Oxford: Elsevier.

Beichner, R. (1994). Testing student interpretation of kinematics graphs. American Journal of Physics, 62, 750-762.

Burns, J.C., Okey, J.R., \& Wise, K.C. (1985). Development of an integrated process skill test: TIPS II. Journal of Research in Science Teaching, 22, 169-177.

Brown, W. (1910). Some experimental results in the correlation of mental abilities. British Journal of Psychology, 3, 296-322.

Chan, C.K.K. (2001). Peer collaboration and discourse patterns in learning from incompatible information. Instructional Science, 29, 443-479.

de Vries, E., Lund, K., \& Baker, M. (2002). Computer-mediated epistemic dialogue: Explanation and argumentation as vehicles for understanding scientific notions. Journal of the Learning Sciences, 11, 63-103.

Dillenbourg, P. (1999). What do you mean by “collaborative learning”? In P. Dillenbourg (Ed.), Collaborative learning: Cognitive and computational approaches (pp. 1-19). Oxford: Elsevier.

Friedler, Y., Nachmias, R., \& Linn, M.C. (1990). Learning scientific reasoning in microcomputer-based laboratories. Journal of Research in Science Teaching, 27, 173-191.

Halloun, I. \& Hestenes, D. (1985). Common sense concepts about motion. American Journal of Physics, 53, 1056-1065.

de Jong, T. \& van Joolingen, W.R. (1998). Scientific discovery learning with computer simulations of conceptual domains. Review of Educational Research, 68, 179-202.

Klahr, D. \& Dunbar, K. (1988). Dual space search during scientific reasoning. Cognitive Science, 12, 1-48.

Kneser, C. \& Ploetzner, R. (2001). Collaboration on the basis of complementary domain knowledge: Observed dialogue structures and their relation to learning success. Learning and Instruction, 11, 53-83.

Kuhn, D., Black, J., Keselman, A., \& Kaplan, D. (2000). The development of cognitive skills to support inquiry learning. Cognition and Instruction, 18, 495-523.

McKenzie, D.L. \& Padilla, M.J. (1986). The construction and validation of the test of graphing in science (TOGS). Journal of Research in Science Teaching, 23, 571-579.

Moschkovich, J.N. (1996). Moving up and getting steeper: Negotiating shared descriptions of linear graphs. Journal of the Learning Sciences, 5, 239-277.

Newman, D., Griffin, P., \& Cole, M. (1989). The construction zone: Working for cognitive change in schools. Cambridge: Cambridge University Press.

Njoo, M.K.H. \& de Jong, T. (1993). Exploratory learning with a computer simulation for control theory: Learning processes and instructional support. Journal of Research in Science Teaching, 30, 821-844. 
Okada, T. \& Simon, H.A. (1997). Collaborative discovery in a scientific domain. Cognitive Science, 21, 109-146.

Ploetzner, R. \& Spada, H. (1992). Analysis-based learning on multiple levels of mental domain representation. In E. de Corte, M. Linn, H. Mandl, \& L. Verschaffel (Eds.), Computerbased learning environments and problem solving (pp. 103-129). Berlin: Springer.

Reimann, P. (1991). Detecting functional relations in a computerized discovery environment. Learning \& Instruction, 1, 45-65.

Rochelle, J. (1992). Learning by collaborating: Convergent conceptual change. Journal of the Learning Sciences, 5, 239-277.

Roth, W.-M. (1995). Affordances of computers in teacher-student interactions: The case of interactive physics. Journal of Research in Science Teaching, 32, 329-347.

Roth, W.-M. \& Roychoudhury, A. (1992). The social construction of scientific concepts or the concept map as conscription device and tool for social thinking in high school science. Science Education, 76, 531-557.

Roth, W.-M., Woszczyna, C., \& Smith, G. (1996). Affordances and constraints of computers in science education. Journal of Research in Science Teaching, 33, 995-1017.

Spearman, C. (1910). Correlation calculated with faulty data. British Journal of Psychology, 3, 271-295.

Swaak, J. \& de Jong, T. (1996). Measuring intuitive knowledge in science: the development of the what-if test. Studies in Educational Evaluation, 22, 341-362.

Teasley, S.D. (1995). The role of talk in children's peer collaborations. Developmental Psychology, 51, 207-220.

Trowbridge, E. \& McDermott, L.C. (1980). Investigation of student understanding of the concept of velocity in one dimension. American Journal of Physics, 48, 1020-1028.

Vahey, P., Enyedy, N., \& Gifford, B. (1999). The probability inquiry environment: A collaborative, inquiry-based simulation environment. Proceedings of the Hawaii International Conference on Systems Sciences (HICSS) 32. Maui, HI.

van Joolingen, W.R. \& de Jong, T. (1997). An extended dual search space model of learning with computer simulations. Instructional Science, 25, 307-334.

van Joolingen, W.R., \& de Jong, T. (2003). SimQuest: Authoring educational simulations. In T. Murray, S. Blessing, \& S. Ainsworth (Eds.), Authoring tools for advanced technology educational software: Toward cost-effective production of adaptive, interactive, and intelligent educational software (pp. 1-31). Dordrecht, The Netherlands: Kluwer.

Vygotsky, L.S. (1962). Thought and language (E. Hanfmann \& G. Vakar, Trans.). Boston: MIT Press. (Original work published in 1934.)

Vygotsky, L.S. (1978). Mind in society: The development of higher psychological processes. In M. Cole, V. John-Steiner, S. Scribner, \& E. Souberman (Eds.), Cambridge, MA: Harvard University Press. (Original work published in 1930.)

Webb, N.M. (1991). Task related verbal interaction and mathematics learning in small groups. Journal for Research in Mathematics Education, 22, 366-389.

Webb, N., Welner, K., \& Zuniga, S. (2001). Short circuits or superconductors? The effects of group composition on high-ability students' science assessment performance. American Educational Research Journal, 39, 943-989.

White, B.Y. \& Frederiksen, J.R. (1990). Causal model progressions as a foundation for intelligent environments. Artificial Intelligence, 42, 99-157.

White, B.Y., Shimoda, T., \& Frederiksen, J. (1999). Enabling students to construct theories of collaborative inquiry and reflective learning: Computer support for metacognitive development. International Journal of Artificial Intelligence in Education, 10, 151-182. 